\title{
Tendências de temperaturas mínimas e máximas do ar no Estado de Minas Gerais
}

\author{
Léo Fernandes Ávila(1), Carlos Rogério de Mello(1), Silvia de Nazaré Monteiro Yanagi(1) \\ e Olívio Bahia Sacramento Neto(2)
}

\begin{abstract}
(1)Universidade Federal de Lavras, Departamento de Engenharia, Caixa Postal 3037, CEP $37200-000$ Lavras, MG, Brasil. E-mail: avilalf@gmail.com, crmello@deg.ufla.br, silviayanagi@deg.ufla.br (2)Instituto Nacional de Pesquisas Espaciais, Centro Nacional de Previsão do Tempo e Clima, Caixa Postal 09, CEP 13418-900 Cachoeira Paulista, SP, Brasil. E-mail: obneto@yahoo.com.br
\end{abstract}

Resumo - O objetivo deste trabalho foi avaliar tendências das temperaturas mínimas e máximas no Estado de Minas Gerais. Foram avaliados dados de 43 municípios, tendo-se considerado as escalas anual e sazonal - janeiro, abril, julho e outubro, que representam os meses centrais de verão, outono, inverno e primavera, respectivamente. Séries históricas de temperaturas mínimas e máximas do ar diárias, com extensão mínima de 30 anos, foram analisadas com base no teste de Mann-Kendall e no uso de regressão linear simples. As tendências foram consideradas significativas somente quando ambas as avaliações foram significativas; neste caso, determinaram-se as taxas de alteração das temperaturas. Houve boa concordância entre os testes de significância para a detecção de tendências nas séries históricas, na maioria dos municípios. Observou-se tendência de elevação das temperaturas mínimas em julho de até $1,5^{\circ} \mathrm{C}$ por década, com tendências generalizadas de aumento na maior parte do estado, em outubro e janeiro, e também na escala anual. Exceções foram verificadas especialmente em municípios de maiores altitudes, em que as temperaturas mínimas diminuíram no inverno. Há predominância de municípios com tendência significativa de aumento de temperaturas, independentemente da escala de avaliação. Tendências de aumento nas temperaturas são esperadas mais comumente no sentido sul-norte do Estado de Minas Gerais.

Termos para indexação: agrometeorologia, aquecimento global, mudanças climáticas, teste de Mann-Kendall, variabilidade climática.

\section{Trends of minimum and maximum air temperatures in the state of Minas Gerais, Brazil}

\begin{abstract}
The objective of this work was to evaluate the trends in minimum and maximum air temperatures in the state of Minas Gerais, Brazil. Data from 43 municipalities were analyzed, considering annual and seasonal scales - January, April, July, and October, which represent the middle months of summer, autumn, winter, and spring, respectively. Historical series of minimum and maximum daily air temperatures, from at least 30 year, were analyzed by the Mann-Kendall test and linear regressions. Trends were considered significant only if both evaluations were significant; in this case, the rates of temperature shift were determined. There was good agreement between the statistical tests for detecting trends in the historical series, for most municipalities. An increasing temperature trend of up to $1.5^{\circ} \mathrm{C}$ per decade was observed for minimum temperatures in July, with generalized increasing trends in the greatest part of the state in January and October, and also in the annual scale. Exceptions were verified mainly in municipalities at higher altitudes, in which minimum air temperatures decreased in winter. There is a predominance of municipalities with significant increasing temperature trend, regardless of the evaluation scale. Increasing temperature trends are expected to be more common in the south to north direction, in the state of Minas Gerais.
\end{abstract}

Index terms: agrometeorology, global warming, climate change, Mann-Kendall test, climatic variability.

\section{Introdução}

Alterações no padrão climático do planeta têm despertado muita atenção pelo potencial de impacto ambiental nos ecossistemas e, especialmente, na agropecuária. $\mathrm{Na}$ região tropical, a economia é fortemente dependente desta atividade, o que aumenta a relevância de um maior conhecimento sobre a ocorrência de eventuais alterações climáticas (Marengo \& Camargo, 2008).

De acordo com os modelos climáticos globais do IPCC (Intergovernmental Panel on Climate Change), até o fim do Século XXI, a temperatura global pode aumentar entre 1,4 e $5,8^{\circ} \mathrm{C}$, o que representaria 
um aquecimento ainda mais acentuado do que o já observado no século 20 (Marcott et al., 2013). Embora o clima varie de forma natural, a velocidade com que tais variações têm ocorrido é preocupante. De acordo com Solomon et al. (2007), grande parte do aumento da temperatura global média, observada no planeta desde meados do século 20, pode ser explicado pelo aumento das concentrações atmosféricas de gases de efeito estufa.

O Brasil apresenta elevada vulnerabilidade aos possíveis efeitos das alterações climáticas, principalmente se considerarmos as projeções atuais de mudança no clima global (Solomon et al., 2007). Assim, é extremamente importante o esforço científico para detectar e quantificar essas alterações, para o planejamento de medidas que possam amenizar seus possíveis efeitos sobre os recursos hídricos, produtividade das lavouras, segurança alimentar e qualidade de vida.

O Estado de Minas Gerais apresenta grande extensão territorial e considerável complexidade climática, com importante influência do relevo sobre a conformação de microclimas. Além disso, sua economia é fortemente dependente de atividades agrícolas, em especial da produção cafeeira, que pode sofrer impactos consideráveis pelas oscilações significativas da temperatura do ar, especialmente na região do Cerrado (Ghini et al., 2008; Camargo, 2010).

A região norte do Estado apresenta clima seco e quente, em contraste com a região sul, que é caracterizada por temperaturas mais amenas e chuvas mais bem distribuídas ao longo do ano (Mello \& Viola, 2013). Além disso, grandes formações montanhosas, com altitudes de até $2.700 \mathrm{~m}$, contrastam com extensos vales, ora intensificando ora inibindo as formações pluviais. A porção leste (Bacia do Rio Doce), que está sujeita à influência oceânica, contrasta com a continentalidade do Triângulo Mineiro e do noroeste do Estado. Tamanha diversidade climática resulta em grande diversidade de paisagens, desde a caatinga, no extremo norte, passando por vastas áreas de Cerrado com diferentes densidades, aos campos de montanhas e às formações de floresta atlântica (Tonietto et al., 2006).

Além de possivelmente o aquecimento global, outros fenômenos têm contribuído para a oscilação climática. Entre eles, merecem destaque a formação de "ilhas de calor" - causadas pelo aumento na urbanização dos municípios (Marengo \& Camargo, 2008) - e, principalmente, as oscilações resultantes do fenômeno Enso (El-Niño Southern Oscillation). No entanto, o efeito desses fenômenos sobre o aumento da temperatura do ar e sobre a ocorrência de eventos extremos de precipitação (Grimm, 2003; Mello et al., 2012) ainda carece de maiores investigações no Brasil (Grimm, 2003).

A complexidade natural do Estado de Minas Gerais, associada às projeções de mudanças climáticas globais, às oscilações naturais do clima, ao crescimento da urbanização e à carência de informações, revela a importância de se estudar o clima de forma regionalizada, como se pretendeu no presente estudo.

O objetivo deste trabalho foi avaliar tendências das temperaturas mínimas e máximas, no Estado de Minas Gerais.

\section{Material e Métodos}

O Estado de Minas Gerais tem área de 582.586 $\mathrm{km}^{2}$ e ocupa aproximadamente $7 \%$ do território brasileiro, entre os paralelos $14^{\circ} 13^{\prime} 57^{\prime \prime}$ e $22^{\circ} 55^{\prime} 47^{\prime \prime} \mathrm{S}$, e os meridianos $39^{\circ} 51^{\prime} 24^{\prime \prime}$ e $51^{\circ} 02^{\prime} 56^{\prime \prime W}$ (Figura 1). Seu regime pluvial apresenta dois períodos bem definidos, um chuvoso, no verão, e outro seco, no inverno. As chuvas concentram-se em seis meses do ano (outubro a abril), e o período entre dezembro e fevereiro é o mais chuvoso. O Estado apresenta alta variabilidade espacial da precipitação média anual, que varia de 700 a $1.000 \mathrm{~mm}$ nas regiões mais secas (norte, nordeste e leste), e de 1.200 a $2.500 \mathrm{~mm}$ nas mais chuvosas (sul, Triângulo, noroeste e Serras do Espinhaço e da Mantiqueira) (Mello \& Viola, 2013).

Quanto à temperatura média, o Estado apresenta influência marcante da latitude e, principalmente, da altitude, em razão da presença de cadeias de montanhas em grande parte de seu território. Assim, os valores médios mensais de temperaturas variam entre $13^{\circ} \mathrm{C}$ (nas regiões mais elevadas, como as das Serras da Mantiqueira e do Espinhaço) e $27^{\circ} \mathrm{C}$ (no norte e leste do Estado), com médias mensais das temperaturas extremas de 4 a $33^{\circ} \mathrm{C}$. Assim, Minas Gerais apresenta expressiva variabilidade de tipos climáticos (Tonnieto et al., 2006). Conforme se observa na Figura 1, ocorrem no Estado, os tipos climáticos $\mathrm{Cwa}$, Cwb, Bsh e Aw, segundo a classificação de Köppen. As estações climatológicas estão identificadas na Tabela 1, com 
suas respectivas coordenadas geográficas, altitudes e séries históricas; sua distribuição espacial encontra-se na Figura 1.

Foram utilizadas séries históricas de temperaturas mínimas e máximas diárias, com período mínimo de observação de 30 anos. Estas séries pertencem à rede de monitoramento do Instituto Nacional de Meteorologia (Inmet) e correspondem a 43 estações climatológicas, no Estado de Minas Gerais (Figura 1 e Tabela 1).

A avaliação da tendência de variação da temperatura mínima e máxima nas séries históricas foi realizada por meio do teste não paramétrico de Mann-Kendall (Mann, 1945; Kendall, 1975) e pela análise de regressão linear simples. As avaliações foram feitas nas escalas sazonal e anual. Na escala sazonal, utilizaram- se os dados de janeiro, abril, julho e setembro, que correspondem aos meses centrais das estações de verão, outono, inverno e primavera, respectivamente. $\mathrm{Na}$ escala anual, as avaliações foram feitas com a série histórica das temperaturas ao longo do ano (valores extremos anuais).

O teste de Mann-Kendall (MK) é frequentemente aplicado para detectar tendências em séries de dados climáticos (Back et al., 2012), bem como para avaliar a ocorrência de mudanças climáticas. Ele é recomendado pela Organização Meteorológica Mundial (Yu et al., 2002) para a avaliação de mudanças climáticas, pois é considerado o método mais adequado para este tipo de análise. Segundo Blain et al. (2011), o teste MK é amplamente utilizado

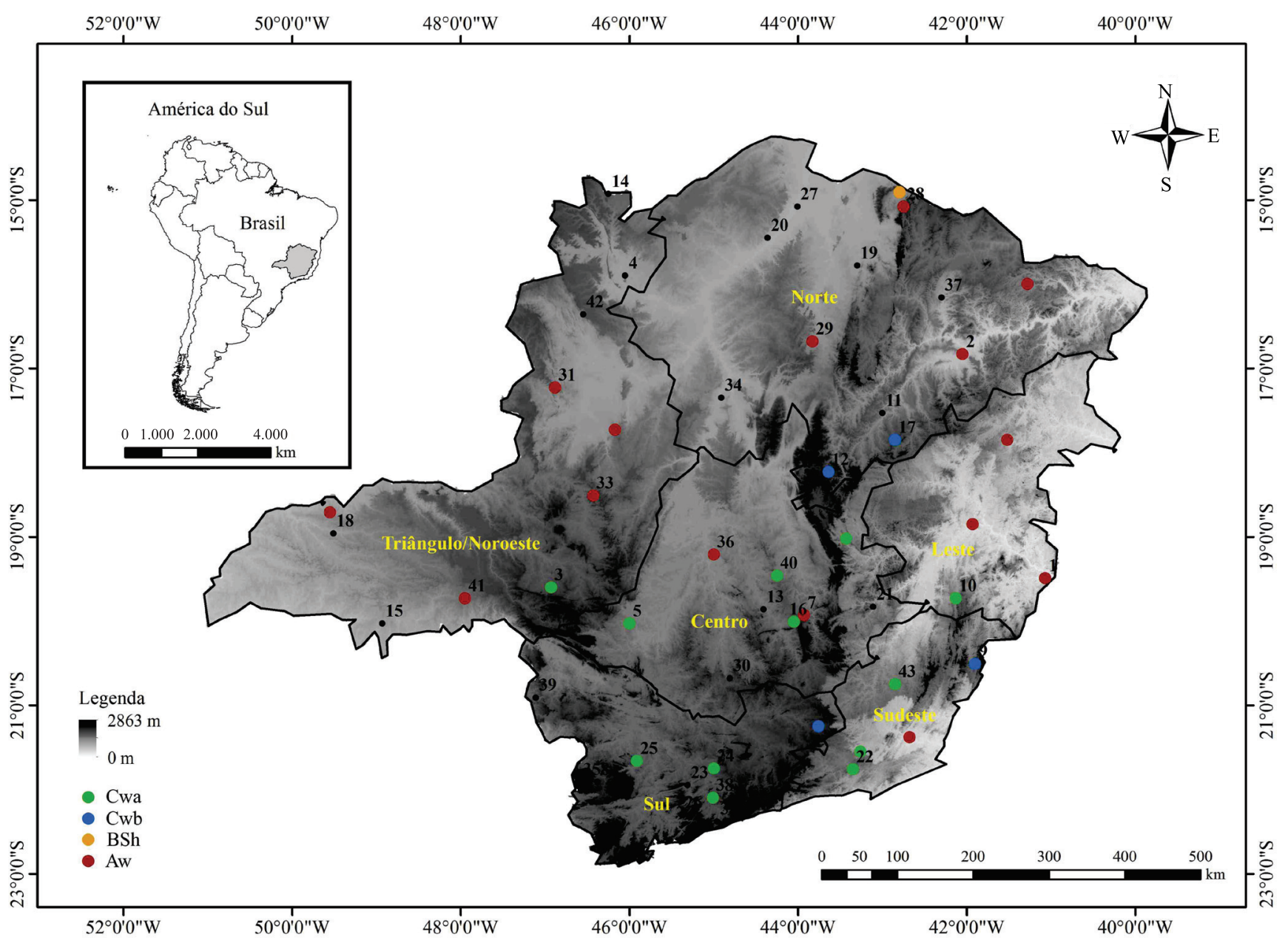

Figura 1. Localização das estações meteorológicas utilizadas no presente estudo (números de 1 a 43, descritos na Tabela 1), com as respectivas classificações climáticas (Köppen), plotadas conforme o modelo digital de elevação do Estado. Fonte: Tonietto et al. (2006) e Instituto Nacional de Meteorologia (2013). 
em estudos de tendências, com séries temporais climatológicas, por apresentar comportamento robusto quanto aos desvios de normalidade e à não estacionariedade dos dados, em comparação a outros métodos. Por se tratar de uma estatística não paramétrica, o teste considera que, no caso de estabilidade da série temporal (Hipótese $\mathrm{H}_{0}$ ), a sucessão de valores ocorre de forma independente, e a distribuição de probabilidade permanece sempre a mesma (série aleatória simples). Assim, valores

Tabela 1. Estações climatológicas utilizadas no estudo, com as respectivas coordenadas geográficas, altitudes e séries históricas aplicadas.

\begin{tabular}{|c|c|c|c|c|c|c|}
\hline $\mathrm{N}^{\circ}$ & Código & Estação & Latitude (graus) & Longitude (graus) & Altitude (m) & Período \\
\hline 1 & 83595 & Aimorés & $-19^{\circ} 29^{\prime}$ & $-41^{\circ} 04^{\prime}$ & 83 & $1972-2009$ \\
\hline 2 & 83442 & Araçuaí & $-16^{\circ} 50^{\prime}$ & $-42^{\circ} 03^{\prime}$ & 289 & 1961-2009 \\
\hline 3 & 83579 & Araxá & $-19^{\circ} 36^{\prime}$ & $-46^{\circ} 56^{\prime}$ & 1.024 & 1963-2009 \\
\hline 4 & 83384 & Arinos & $-15^{\circ} 54^{\prime}$ & $-46^{\circ} 03^{\prime}$ & 519 & 1976-2009 \\
\hline 5 & 83582 & Bambuí & $-20^{\circ} 02^{\prime}$ & $-46^{\circ} 00^{\prime}$ & 661 & 1961-2009 \\
\hline 6 & 83689 & Barbacena & $-21^{\circ} 15^{\prime}$ & $-43^{\circ} 46^{\prime}$ & 1.126 & 1961-2009 \\
\hline 7 & 83587 & Belo Horizonte & $-19^{\circ} 56^{\prime}$ & $-43^{\circ} 56^{\prime}$ & 915 & 1961-2009 \\
\hline 8 & 83681 & Caldas & $-21^{\circ} 55^{\prime}$ & $-46^{\circ} 23^{\prime}$ & 1.150 & 1961-2009 \\
\hline 9 & 83639 & Caparaó & $-20^{\circ} 31^{\prime}$ & $-41^{\circ} 54^{\prime}$ & 843 & 1973-2009 \\
\hline 10 & 83592 & Caratinga & $-19^{\circ} 44^{\prime}$ & $-42^{\circ} 08^{\prime}$ & 610 & 1961-2009 \\
\hline 11 & 83485 & Carbonita & $-17^{\circ} 32^{\prime}$ & $-43^{\circ} 00^{\prime}$ & 736 & 1981-2009 \\
\hline 12 & 83538 & Diamantina & $-18^{\circ} 14^{\prime}$ & $-43^{\circ} 38^{\prime}$ & 1.296 & 1966-2009 \\
\hline 13 & 83581 & Florestal & $-19^{\circ} 52^{\prime}$ & $-44^{\circ} 25^{\prime}$ & 749 & 1961-2009 \\
\hline 14 & 83334 & Formoso & $-14^{\circ} 56^{\prime}$ & $-46^{\circ} 15^{\prime}$ & 840 & 1976-2009 \\
\hline 15 & 83574 & Frutal & $-20^{\circ} 02^{\prime}$ & $-48^{\circ} 56^{\prime}$ & 544 & 1961-2009 \\
\hline 16 & 83632 & Ibirité & $-20^{\circ} 01^{\prime}$ & $-44^{\circ} 03^{\prime}$ & 815 & 1961-2009 \\
\hline 17 & 83488 & Itamarandiba & $-17^{\circ} 51^{\prime}$ & $-42^{\circ} 51^{\prime}$ & 1.097 & $1962-2009$ \\
\hline 18 & 83521 & Ituiutaba & $-18^{\circ} 58^{\prime}$ & $-49^{\circ} 31^{\prime}$ & 560 & 1980-2009 \\
\hline 19 & 83395 & Janaúba & $-15^{\circ} 47^{\prime}$ & $-43^{\circ} 18^{\prime}$ & 516 & $1977-2010$ \\
\hline 20 & 83386 & Januária & $-15^{\circ} 27^{\prime}$ & $-44^{\circ} 22^{\prime}$ & 474 & $1961-2010$ \\
\hline 21 & 83591 & João Monlevade & $-19^{\circ} 50^{\prime}$ & $-43^{\circ} 07^{\prime}$ & 860 & 1961-2010 \\
\hline 22 & 83692 & Juiz de Fora & $-21^{\circ} 46^{\prime}$ & $-43^{\circ} 21^{\prime}$ & 940 & 1961-2009 \\
\hline 23 & 83032 & Lambari & $-21^{\circ} 57^{\prime}$ & $-45^{\circ} 19^{\prime}$ & 878 & 1976-2009 \\
\hline 24 & 83687 & Lavras & $-21^{\circ} 45^{\prime}$ & $-45^{\circ} 00^{\prime}$ & 919 & 1961-2009 \\
\hline 25 & 83683 & Machado & $-21^{\circ} 40^{\prime}$ & $-45^{\circ} 55^{\prime}$ & 873 & 1961-2009 \\
\hline 26 & 83015 & Maria da Fé & $-22^{\circ} 18^{\prime}$ & $-45^{\circ} 23^{\prime}$ & 1.276 & 1976-2009 \\
\hline 27 & 83389 & Muzambinho & $-15^{\circ} 05^{\prime}$ & $-44^{\circ} 01^{\prime}$ & 452 & 1976-2009 \\
\hline 28 & 83388 & Monte Azul & $-15^{\circ} 05^{\prime}$ & $-42^{\circ} 45^{\prime}$ & 604 & 1974-2009 \\
\hline 29 & 83437 & Montes Claros & $-16^{\circ} 41^{\prime}$ & $-43^{\circ} 50^{\prime}$ & 646 & 1961-2009 \\
\hline 30 & 83637 & Oliveira & $-20^{\circ} 41^{\prime}$ & $-44^{\circ} 49^{\prime}$ & 967 & 1962-2009 \\
\hline 31 & 83479 & Paracatu & $-17^{\circ} 14^{\prime}$ & $-46^{\circ} 53^{\prime}$ & 712 & 1973-2009 \\
\hline 32 & 83737 & Passa quatro & $-22^{\circ} 23^{\prime}$ & $-44^{\circ} 58^{\prime}$ & 920 & 1961-2009 \\
\hline 33 & 83531 & Patos de Minas & $-18^{\circ} 31^{\prime}$ & $-46^{\circ} 26^{\prime}$ & 940 & 1961-2009 \\
\hline 34 & 83483 & Pirapora & $-17^{\circ} 21^{\prime}$ & $-44^{\circ} 55^{\prime}$ & 505 & 1961-2009 \\
\hline 35 & 83681 & Poços de Caldas & $-21^{\circ} 55^{\prime}$ & $-46^{\circ} 29^{\prime}$ & 1.150 & 1961-2009 \\
\hline 36 & 83570 & Pompeu & $-19^{\circ} 13^{\prime}$ & $-45^{\circ} 00^{\prime}$ & 691 & 1973-2009 \\
\hline 37 & 83441 & Salinas & $-16^{\circ} 10^{\prime}$ & $-42^{\circ} 18^{\prime}$ & 471 & 1961-2009 \\
\hline 38 & 83736 & São Lourenço & $-22^{\circ} 06^{\prime}$ & $-45^{\circ} 01^{\prime}$ & 953 & 1961-2009 \\
\hline 39 & 83631 & São Sebastião do Paraíso & $-20^{\circ} 55^{\prime}$ & $-47^{\circ} 07^{\prime}$ & 820 & 1961-2009 \\
\hline 40 & 83586 & Sete Lagoas & $-19^{\circ} 28^{\prime}$ & $-44^{\circ} 15^{\prime}$ & 732 & 1961-2009 \\
\hline 41 & 83577 & Uberaba & $-19^{\circ} 44^{\prime}$ & $-47^{\circ} 57^{\prime}$ & 737 & 1961-2009 \\
\hline 42 & 83428 & Unaí & $-16^{\circ} 22^{\prime}$ & $-46^{\circ} 33^{\prime}$ & 460 & 1978-2009 \\
\hline 43 & 83642 & Viçosa & $-20^{\circ} 45^{\prime}$ & $-42^{\circ} 51^{\prime}$ & 690 & 1961-2009 \\
\hline
\end{tabular}

Fonte: Instituto Nacional de Meteorologia (2013). 
positivos do coeficiente de Mann-Kendall $(\mathrm{MK}>0)$ indicam tendência de crescimento da variável em estudo, enquanto valores negativos $(\mathrm{MK}<0)$ indicam tendência de diminuição. Considerandose a probabilidade de $5 \%$, a Tabela de $\mathrm{Z}$ fornece $Z_{0,975}=1,96$. Assim, se a estatística do teste de Mann Kendall (MK) for superior a 1,96 - ou seja, $|\mathrm{MK}|>\mathrm{Z}_{1-\alpha / 2}$ - rejeita-se a hipótese $\mathrm{H}_{0}$ e aceita-se a existência de tendência significativa na série de dados de temperatura.

A análise de regressão foi baseada na verificação do coeficiente angular da reta ajustada aos dados. No caso de valores estatisticamente significativos, há indício de possíveis alterações nas séries de temperaturas mínima e máxima. A análise consistiu da determinação do intervalo de confiança do coeficiente angular e, no caso de este intervalo não incluir o valor zero, considerou-se a tendência significativa no nível de confiança avaliado. Desta forma, se o coeficiente angular da equação da reta for positivo, ele sinaliza tendência significativa de aumento da temperatura e, se negativo, ele sinaliza tendência de redução (Back et al., 2012). Para o teste de significância da regressão, adotou-se o nível de confiança de 5\%, tendo-se aplicado o teste t de Student (Wilks, 1995) para a comparação com o valor crítico da Tabela (teste bilateral), com $\mathrm{n}$ - 2 graus de liberdade. $\mathrm{O}$ teste $\mathrm{t}$ avalia se o coeficiente angular da regressão é significantemente diferente de zero, o que indicaria a presença de tendência linear (Longobardi \& Villani, 2010).

A análise de regressão também foi utilizada para estimar as taxas de acréscimo ou decréscimo das temperaturas mínimas e máximas nas tendências decenais (Back et al., 2012).

O teste de Mann-Kendall e a análise de regressão são complementares: o primeiro avalia a significância da tendência, e o segundo, o grau de inclinação da reta e as projeções decenais, isto é, as taxas de variação das temperaturas mínimas e máximas. Os dados somente foram plotados para municípios que apresentaram tendências significativas (positivas ou negativas) atestadas por ambos os métodos, conforme sugerido por Minuzzi et al. (2010).

Para as análises estatísticas, utilizou-se o programa XLSTAT Versão 2011.3.02 e, para a confecção dos mapas, utilizaram-se os aplicativos ArcMap, ArcCatalog e ArcToolbox, do programa ArcGIS Desktop 10.1.

\section{Resultados e Discussão}

Na Figura 2, estão apresentados os resultados dos testes estatísticos plotados sobre o Estado de Minas Gerais e as taxas significativas de variação das temperaturas mínimas, detectadas simultaneamente pelo teste de Mann-Kendall e pela análise de regressão, na escala sazonal. Em janeiro (verão) e outubro (primavera), a maioria dos municípios $(86,7 \%)$ mostrou tendência significativa de aumento nas temperaturas mínimas. Minuzzi et al. (2010), no entanto, ao analisar tendências das temperaturas mínimas de nove municípios do Estado (série histórica de 1961 a 2004), constataram aumento significativo da temperatura mínima apenas em Viçosa, em 7 dos 9 meses avaliados, principalmente na década de 1990 .

Em janeiro, dos 26 municípios que mostraram tendência significativa de aumento das temperaturas mínimas, 21 apresentaram taxas de até $0,5^{\circ} \mathrm{C}$ por década, quatro com taxas entre 0,5 e $1,0^{\circ} \mathrm{C}$ por década, e apenas um (Maria da Fé) com taxa superior a $1,0^{\circ} \mathrm{C}$ por década (Figura 2). Nesse mês, somente o município de Juiz de Fora apresentou tendência significativa de diminuição das temperaturas mínimas, com taxas de até $-0,5^{\circ} \mathrm{C}$ por década. Martinez et al. (2012), ao avaliar o comportamento das temperaturas mínimas no Estado da Flórida, nos EUA, que apresenta condições climáticas similares às do Estado de Minas Gerais, observaram tendência predominantemente positiva nas séries históricas, especialmente entre 1970 e 2009, período similar ao analisado no presente estudo.

Em outubro, dos 26 municípios que mostraram tendência de aquecimento, em 21 foram identificadas taxas de até $0,5^{\circ} \mathrm{C}$ por década e, em cinco, taxas de 0,5 a $1,0^{\circ} \mathrm{C}$ por década. Apenas São Lourenço apresentou tendência de redução das temperaturas mínimas, com taxas de até $-0,5^{\circ} \mathrm{C}$ por década.

Nos meses de abril (outono) e julho (inverno), o número de estações meteorológicas que apresentaram tendência de aumento das temperaturas mínimas (30,3 e $37,2 \%$, respectivamente) foi consideravelmente menor do que em janeiro e outubro. Martinez et al. (2012) observaram resultado similar no Estado da Flórida (EUA), onde a predominância de aumento das temperaturas (máximas e mínimas) foi observada durante o verão; contudo, os autores observaram aumentos significativos generalizados, tanto das mínimas quanto das máximas. Para abril, caracteristi- 
camente um mês de transição climática em Minas Gerais, 23,3\% dos municípios apresentaram tendência de aumento, com taxas de até $0,5^{\circ} \mathrm{C}$ por década, enquanto $7 \%$ com taxas entre 0,5 e $1,0^{\circ} \mathrm{C}$ por década.
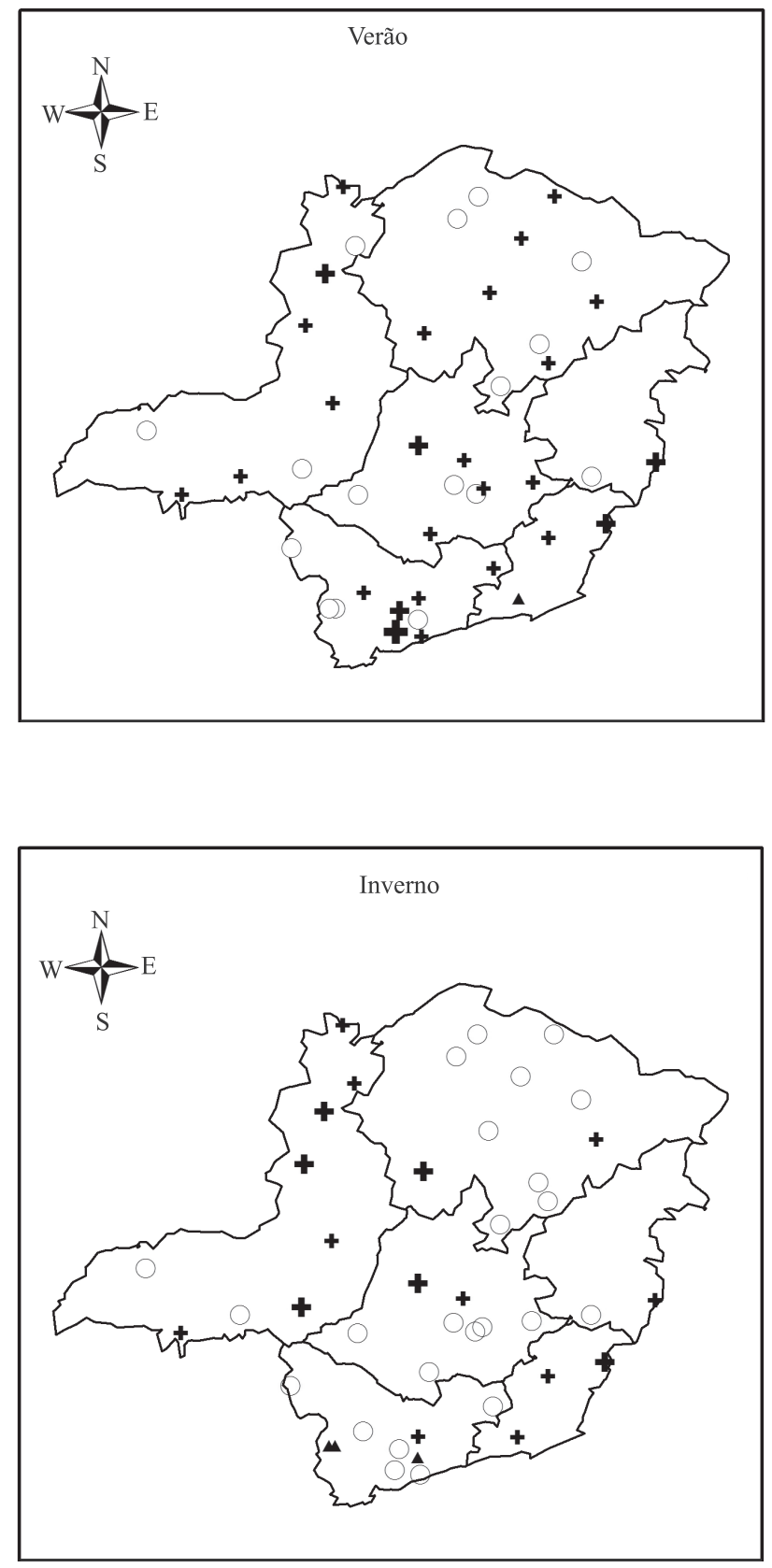

Somente o Município de São Sebastião do Paraíso apresentou tendência de redução das temperaturas mínimas em abril, com taxas de até $-0,5^{\circ} \mathrm{C}$ por década.
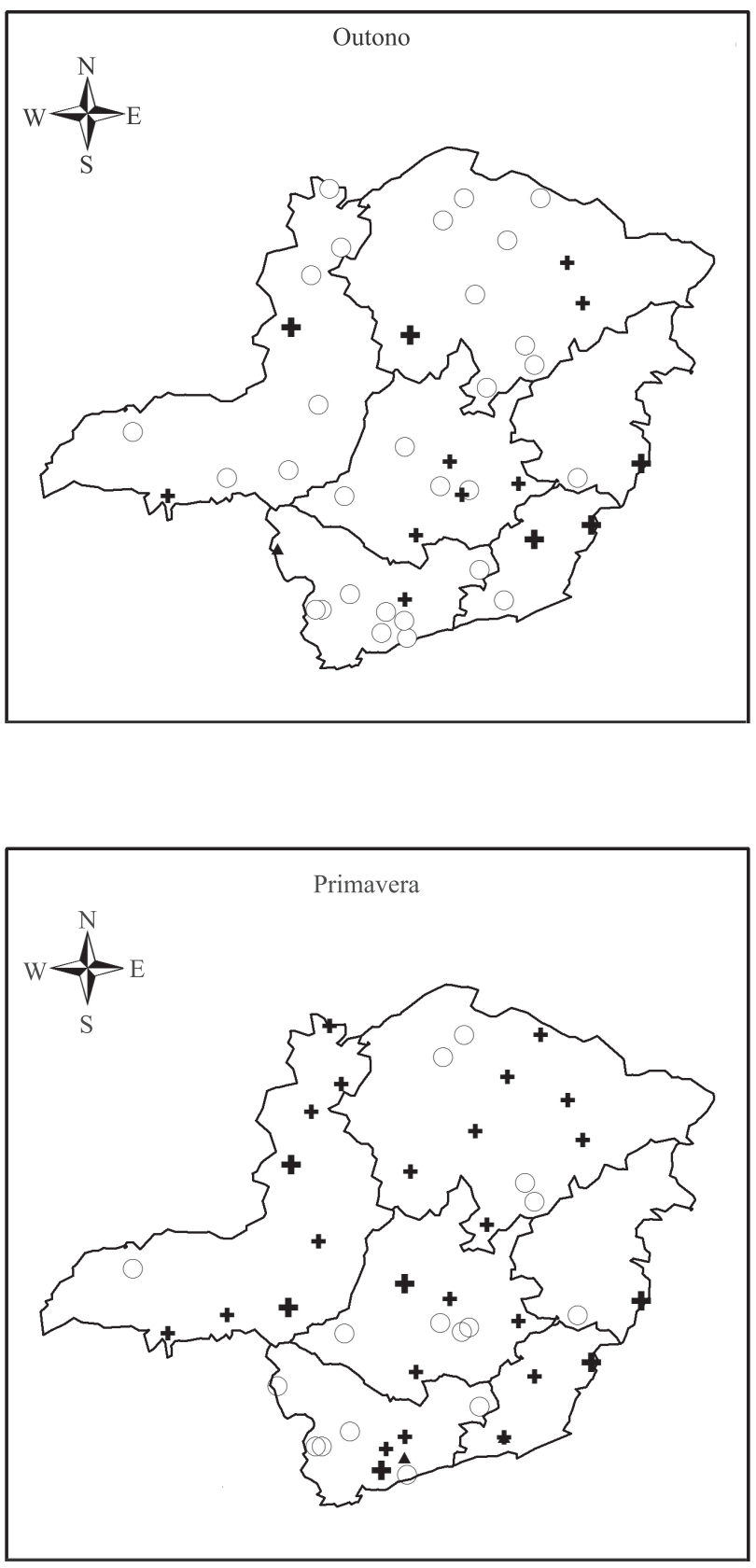

${ }^{\circ} \mathrm{C}$ por década

$+0,0-0,5+0,5-1,0 \Delta 0,0-0,5 \bigcirc$ Sem tendência

Figura 2. Taxas de variação da temperatura mínima, para o período de 1961 a 2009, nos municípios avaliados do Estado de Minas Gerais. Foram consideradas somente as taxas simultaneamente significativas pelos testes de Mann-Kendall e $t$ de Student (regressão linear). 
Em julho, 25,6\% dos municípios apresentaram aumento a taxas de até $0,5^{\circ} \mathrm{C}$ por década, e $11,6 \%$, a taxas entre 0,5 a $1,0^{\circ} \mathrm{C}$ por década. $\mathrm{O}$ mês de julho teve três municípios com tendência de queda da temperatura mínima, com taxas de até $-0,5^{\circ} \mathrm{C}$ por década: Caldas, Poços de Caldas e São Lourenço, todos localizados na região sul do Estado. Merece destaque o fato de que municípios com tendência significativa de redução da temperatura mínima ou que não tenham apresentado alteração significativa sofrem influência marcante da altitude em suas condições climáticas (Tabela 1).

Neste tipo de análise, é importante observar que, além das mudanças climáticas globais, fatores como urbanização ("ilhas de calor"), mudanças da localização dos postos de observação e, principalmente, efeitos de fenômenos globais de oscilação interdecadais desempenham papel marcante na temperatura dos municípios. Minuzzi et al. (2010) relatam que, aparentemente, esses fenômenos de oscilação interdecadais são os principais responsáveis pelo aumento da temperatura do ar em Minas Gerais. Blain et al. (2009), ao analisar o comportamento da temperatura mínima em seis localidades do Estado de São Paulo, observaram que três delas não apresentaram qualquer tendência significativa nesta série. Os autores concluíram, portanto, que fatores de escala local, como urbanização e áreas montanhosas, possivelmente se sobrepõem a fatores de escala global como os principais responsáveis por alterações nos valores médios da temperatura mínima.

Quanto às taxas de variação das temperaturas máximas, na escala sazonal (Figura 3), o número de municípios com tendência de aumento (seis municípios) foi significativamente menor do que na análise das temperaturas mínimas. Apenas o Município de Juiz de Fora apresentou tendência significativa de redução das temperaturas máximas, com taxas de até $-0,5^{\circ} \mathrm{C}$ por década. É importante lembrar que esta mesma estação climatológica também apresentou tendência de redução das temperaturas mínimas, para o mês de janeiro (Figura 2).

Em abril, 22 municípios apresentaram tendência de aumento das temperaturas máximas: 18 com taxas de até $0,5^{\circ} \mathrm{C}$ por década, e quatro com taxas entre 0,5 a $1,0^{\circ} \mathrm{C}$ por década (Figura 3). Em julho, foram 26 municípios: 23 com taxas de até $0,5^{\circ} \mathrm{C}$ por década, e três com taxas entre 0,5 a $1,0^{\circ} \mathrm{C}$ por década. $\mathrm{O}$ mês de outubro foi o que apresentou maior número de municípios com tendência de aumento das temperaturas máximas: 30 com tendência significativa de aumento (13 com taxas de até $0,5^{\circ} \mathrm{C}$ por década, e $17 \mathrm{com}$ taxas entre 0,5 a $1,0^{\circ} \mathrm{C}$ por década). Minuzzi et al. (2010) chegaram à mesma conclusão quanto à temperatura máxima, em que setembro e dezembro figuraram como os meses de aumentos mais significativos. Minuzzi et al. (2011) também relataram maior número de tendências positivas significativas na primavera, no Estado do Paraná.

Em janeiro, apenas seis municípios apresentaram tendência de aumento das temperaturas máximas: dois com taxas de até $0,5^{\circ} \mathrm{C}$ por década, e quatro com taxas entre 0,5 e $1,0^{\circ} \mathrm{C}$ por década. Este número foi baixo, se comparado à análise com temperaturas mínimas para o mês, que mostrou 26 municípios com tendência significativa de aumento da temperatura. Blain (2009) observou que a temperatura máxima média anual apresentou tendência de aumento significativo em dois municípios no Estado de São Paulo e, em outros quatro, não foram observadas alterações significativas das temperaturas.

É interessante ressaltar que o número de municípios com tendência de redução das temperaturas máximas foi menor do que na análise com as temperaturas mínimas, em que apenas um município apresentou tendência de redução das temperaturas máximas, enquanto 11 municípios apresentaram tendência de redução das mínimas.

$\mathrm{Na}$ escala anual, o número de municípios com tendência de aumento, tanto nas temperaturas mínimas como nas máximas, foi maior do que o observado na escala sazonal (Figura 4). Nessa escala, 27 municípios apresentaram tendência significativa de aumento das temperaturas mínimas: $24 \mathrm{com}$ taxas de até $0,5^{\circ} \mathrm{C}$ por década e três com taxas entre 0,5 a $1^{\circ} \mathrm{C}$ por década. Quanto às máximas, 37 municípios apresentaram tendência de aumento: $36 \mathrm{com}$ taxas de até $0,5^{\circ} \mathrm{C}$ por década e apenas 1 (Maria da Fé) com taxa entre 0,5 e $1{ }^{\circ} \mathrm{C}$ por década.

Em termos espaciais, o número de municípios com alterações significativas das temperaturas médias é maior à medida que se desloca para as regiões central, norte, nordeste e noroeste do Estado de Minas Gerais, indício de que estas regiões têm apresentado maior tendência de aquecimento nos últimos 40 anos.

Destaca-se que, dos municípios que apresentaram redução das temperaturas mínimas, na escala sazonal, apenas três (Caldas, Poços de Caldas e São Lourenço) 
também apresentaram redução na escala anual. Esses municípios apresentaram tendência de redução à época de menores registros de temperaturas, ou seja, no mês de julho (inverno), corroborando, assim, a ligeira tendência de redução na escala anual, nesses municípios.
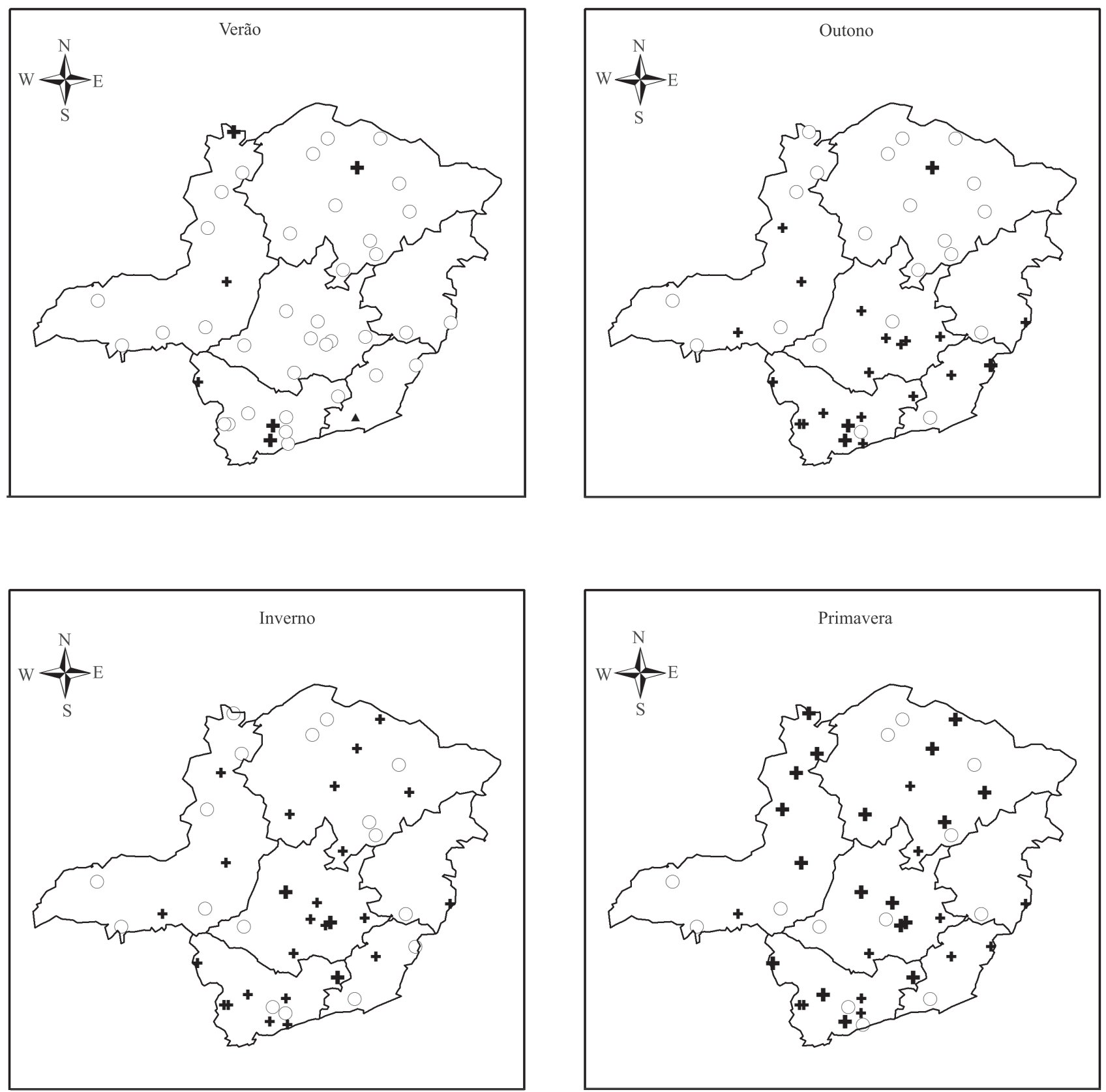

$$
\begin{aligned}
& { }^{\circ} \mathrm{C} \text { por década } \\
& +0,0-0,5+0,5-1,0 \Delta 0,0--0,5 \bigcirc \text { Sem tendência }
\end{aligned}
$$

Figura 3. Taxas de variação da temperatura máxima, para o período de 1961 a 2009, nos municípios avaliados do Estado de Minas Gerais. Foram consideradas somente as taxas simultaneamente significativas pelos testes de Mann-Kendall e t de Student (regressão linear). 

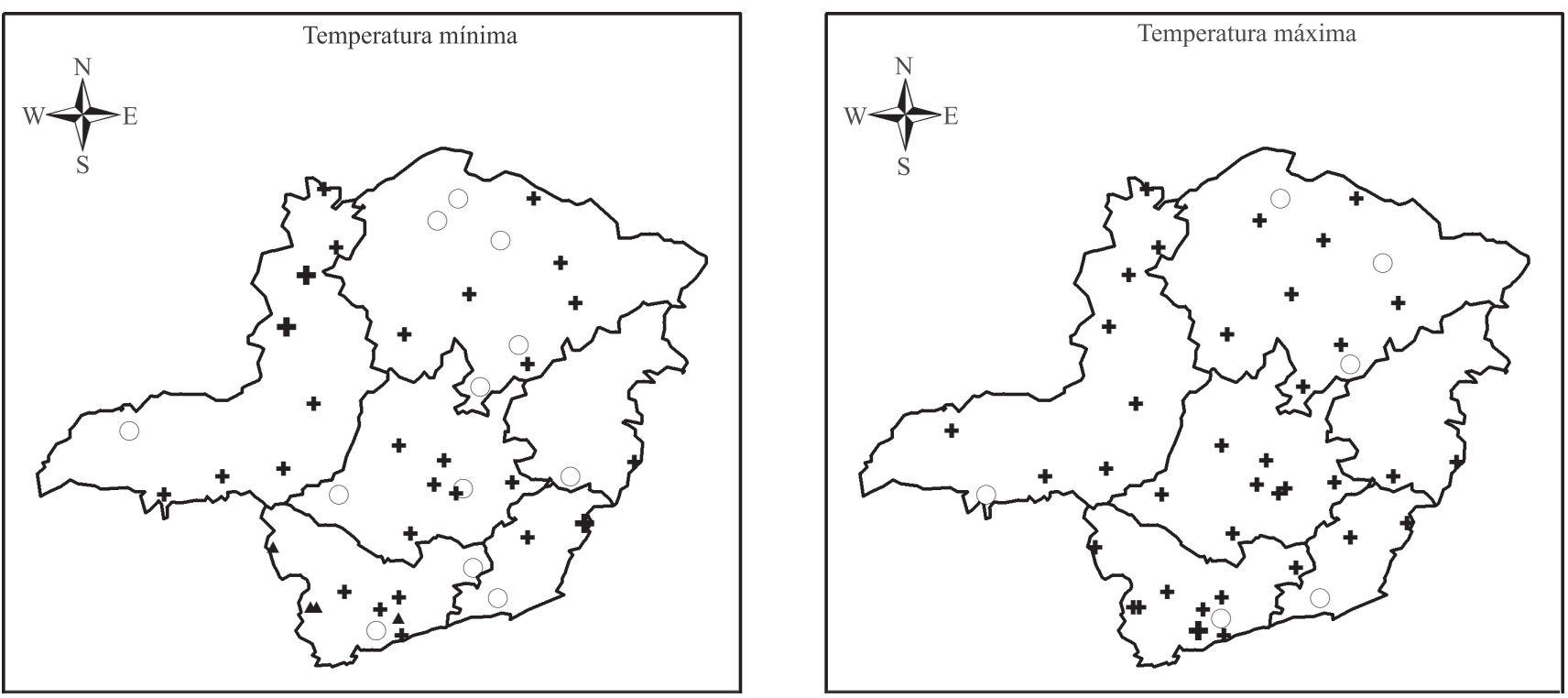

${ }^{\circ} \mathrm{C}$ por década
$+0,0-0,5+0,5-1,0 \Delta 0,0--0,5 \bigcirc$ Sem tendência

Figura 4. Taxas de variação significativa das temperaturas mínima (A) e máxima (B), para o período de 1961 a 2009, na escala anual, para os municípios avaliados do Estado de Minas Gerais. Foram consideradas somente as taxas simultaneamente significativas pelos testes de Mann-Kendall e t de Student (regressão linear).

\section{Conclusões}

1. Há ampla predominância de tendências significativas de aumento das temperaturas mínimas e, em menor instância, das máximas, no Estado de Minas Gerais.

2. As tendências de aumento das temperaturas mínimas e máximas são mais acentuadas no mês de outubro, para a maioria dos municípios de Minas Gerais.

3. Tendência significativa de redução das temperaturas mínimas somente foi observada em julho, geralmente em municípios com maior altitude.

4. As temperaturas tendem a aumentar no sentido sul-norte, no Estado de Minas Gerais.

\section{Agradecimentos}

À Coordenadoria de Aperfeiçoamento de Pessoal de Nível Superior (Capes), por concessão de bolsa (Processo 3002/2011) e à Fundação de Amparo à Pesquisa do Estado de Minas Gerais (Fapemig), pelo apoio financeiro (Projeto 068/2012).

\section{Referências}

BACK, A.J.; BRUNA, E.D.; VIEIRA, H.J. Tendências climáticas e produção de uva na região dos Vales da Uva Goethe. Pesquisa Agropecuária Brasileira, v.47, p.497-504, 2012. DOI: 10.1590/ S0100-204X2012000400004.

BLAIN, G.C. Considerações estatísticas relativas a seis séries mensais de temperatura do ar da Secretaria de Agricultura e Abastecimento do Estado de São Paulo. Revista Brasileira de Meteorologia, v.26, p.279-296, 2011. DOI: 10.1590/ S0102-77862011000200012.

BLAIN, G.C. Séries anuais de temperatura máxima média do ar no Estado de São Paulo: variações e tendências climáticas. Revista Brasileira de Meteorologia, v.25, p.114-124, 2009. DOI: 10.1590/ S0102-77862010000100010.

BLAIN, G.C.; PICOLI, M.C.A.; LULU, J. Análises estatísticas das tendências de elevação nas séries anuais de temperatura mínima do ar no Estado de São Paulo. Bragantia, v.68, p.807-815, 2009. DOI: 10.1590/S0006-87052009000300030.

CAMARGO, M.B.P. de. The impact of climatic variability and climate change on arabic coffee crop in Brazil. Bragantia, v.69, p.239-247, 2010. DOI: 10.1590/S0006-87052010000100030.

GHINI, R.; HAMADA, E.; PEDRO JÚNIOR, M.J.; MARENGO J.A.; GONÇALVES, R.R. do V. Risk analysis of climate change on coffee nematodes and leaf miner in Brazil. Pesquisa Agropecuária Brasileira, v.43, p.187-194, 2008. DOI: 10.1590/ S0100-204X2008000200005. 
GRIMM, A.M. The El-Niño impact on the summer monsoon in Brazil: regional processes versus remote influences. Journal of Climate, v.16, p.263-280, 2003. DOI: 10.1175/1520-0442(2003)016<0263:TENIOT>2.0.CO;2.

INSTITUTO NACIONAL DE METEOROLOGIA. Instituto Nacional de Meteorologia. Disponível em: <http://www.inmet. gov.br/portal/>. Acesso em: 11 nov. 2013.

KENDALL, M.G. Rank correlation methods. London: Charles Griffin, 1975. 210p.

LONGOBARDI, A.; VILLANI, P. Trend analysis of annual and seasonal rainfall time series in the Mediterranean area. International Journal of Climatology, v.30, p.1538-1546, 2010. DOI: $10.1002 /$ joc. 2001 .

MANN, H.B. Nonparametric test against trend. Econometrika, v.13, p.245-259, 1945. DOI: 10.2307/1907187.

MARCOTT, S.A.; SHAKUN, J.D.; CLARK, P.U.; MIX, A.C. A reconstruction of regional and global temperature for the past 11.300 years. Science, v.339, p.1198-1201, 2013. DOI: 10.1126/ science. 1228026 .

MARENGO, J.A.; CAMARGO, C.C. Surface air temperature trends in Southern Brazil for 1960-2002. International Journal of Climatology, v.28, p.893-904, 2008. DOI: 10.1002/joc.1584.

MARTINEZ, C.J.; MALESKI, J.J.; MILLER, M.F. Trends in precipitation and temperature in Florida, USA. Journal of Hydrology, v.452, p.259-281, 2012. DOI: 10.1016/j. jhydrol.2012.05.066.

MELLO, C.R. de; NORTON, L.D.; CURI, N.; YANAGI, S.N.M. Sea surface temperature (SST) and rainfall erosivity in the Upper
Grande River Basin, Southeast Brazil. Ciência e Agrotecnologia, v.36, p.53-59, 2012. DOI: 10.1590/S1413-70542012000100007.

MELLO, C.R. de; VIOLA, M.R. Mapeamento de chuvas intensas no Estado de Minas Gerais. Revista Brasileira de Ciência do Solo, v.37, p.37-44, 2013. DOI: 10.1590/S0100-06832013000100004.

MINUZZI, R.B.; CARAMORI, P.H.; BORROZINO, E. Tendências na variabilidade climática sazonal e anual das temperaturas máxima e mínima do ar no Estado do Paraná. Bragantia, v.70, p.471-479, 2011. DOI: 10.1590/S0006-87052011000200031.

MINUZZI, R.B.; VIANELLO, R.L.; SEDIYAMA, G.C. Oscilações climáticas em Minas Gerais. Revista Brasileira de Meteorologia, v.25, p.227-236, 2010. DOI: 10.1590/S0102-77862010000200007.

SOLOMON, S.; QIN, D.; MANNING, M.; CHEN, Z.; MARQUIS, M.; AVERYT, K.B.; TIGNOR, M.; MILLER, H.L. (Ed.). Climate change 2007: the physical science basis: contribution of Working Group I to the Fourth Assessment Report of the Intergovernmental Panel on Climate Change. Cambridge: Cambridge University, 2007. 996p.

TONIETTO, J.; VIANELLO, R.L.; REGINA, M.A. Caracterização macroclimática e potencial enológico de diferentes regiões com vocação vitícola de Minas Gerais. Informe Agropecuário, v.27, p.32-55, 2006.

WILKS, D.S. Statistical methods in the atmospheric sciences. San Diego: Academic Press, 1995. 467p.

YU, P.S.; TAO, T.C.; CHOU, C.C. Effects of climate change on evapotranspiration from paddy fields in Southern Taiwan. Climatic Change, v.54, p.165-179, 2002. DOI: 10.1023/A:1015764831165.

Recebido em 18 de fevereiro de 2014 e aprovado em 28 de março de 2014

Pesq. agropec. bras., Brasília, v.49, n.4, p.247-256, abr. 2014

DOI: $10.1590 / \mathrm{S} 0100-204 X 2014000400002$ 